\title{
Star cluster survival in dark matter halos: An old cluster in Eridanus II?
}

\author{
Jairo A. Alzate, ${ }^{1 \star}$ Verónica Lora, ${ }^{1}$ Gustavo Bruzual, ${ }^{1}$ Luis Lomelí-Núñez, ${ }^{2}$ \\ Bernardo Cervantes Sodi ${ }^{1}$ \\ ${ }^{1}$ Instituto de Radioastronomía y Astrofísica, UNAM, Campus Morelia, Michoacán, C.P. 58089, México \\ ${ }^{2}$ Instituto Nacional de Astrofísica, Optica y Electrónica, Calle Luis Enrique Erro, No 1, Tonantzintla, Puebla, C.P. 72840, México
}

Accepted XXX. Received YYY; in original form ZZZ

\begin{abstract}
The star formation history and the internal dynamics of Milky Way satellite galaxies are often complicated. In the last years, a substantial fraction of the known faint dwarf satellites have been studied. Some of them show embedded stellar substructures, such as star clusters and even globular star clusters. In this work we study Eridanus II, a dwarf spheroidal satellite which hosts a star cluster, using published and archival data from the Hubble Space Telescope Advanced Camera for Surveys. We employ a Bayesian hierarchical method to infer the star formation history of Eridanus II. We find that the bulk of the stars in Eridanus II are very old $\left(13.5_{-1}^{+0.5} \mathrm{Gyr}\right)$ and quite metal poor $(Z=0.00001)$. We do not find any evidence of the presence of an intermediate age or young population in Eri II. We cannot date the embedded star cluster as a separate entity, but we find it likely that the cluster has a similar age and metallicity as the bulk of the stars in Eri II.

The existence of an old star cluster in a dark matter dominated old metal poor dwarf galaxy is of major importance to cast light on the dark matter distribution within dwarf galaxies. The existence of intermediate age stars is required by the recent detection of carbon stars in Eri II. Since no recent star formation is detected, blue-straggler fusions of lower mass stars are the most likely origin of the carbon star progenitors.
\end{abstract}

Key words:

galaxies: dwarf - galaxies: star cluster: individual: Eridanus II cluster - galaxies: star formation - galaxies: statistics - galaxies:stellar content

\section{INTRODUCTION}

Dwarf galaxies are the most numerous galaxies in the Universe. They come in different flavors, dwarf spheroidals (dSphs) being just one of them. Because dSphs lack gas and most of their stars are old (age > $10 \mathrm{Gyr}$, Grebel \& Gallagher 2004), they were once considered simple stellar populations (SSPs). As better observations have been obtained and more research accomplished, it has become clear that these galaxies contain more than meets the eye. To study dwarf galaxies it is a natural choice to start with the satellite galaxies of the Milky Way (MW), which by their proximity can be resolved into individual stars and their evolutionary history traced in great detail. Muñoz et al. (2018) carried out a wide-field imaging survey of the MW satellites in the outer halo (Galactocentric distance $>25$ $\mathrm{kpc}$ ) and derived structural parameters for 58 galaxies, $75 \%$ of the known outer halo satellites.

One particularly interesting MW satellite is the dSph galaxy Eridanus II (Eri II hereafter, shown in Fig. 1; Bechtol et al. 2015;

^ E-mail: j.alzate@irya.unam.mx
Koposov et al. 2015; Crnojević et al. 2016; Li et al. 2017; Zoutendijk et al. 2020; Simon et al. 2021; Gallart et al. 2021) ${ }^{1}$. At a Galactocentric distance of $368 \pm 17 \mathrm{kpc}$ (distance modulus $=22.83$ Crnojević et al. 2016), Eri II is beyond the $300 \mathrm{kpc}$ virial radius of the MW. Using Magellan/IMACS spectroscopy, Li et al. (2017) measured a velocity dispersion of $6.9_{-0.9}^{+1.2} \mathrm{~km} \mathrm{~s}^{-1}$ for Eri II. For a velocity dispersion supported system in equilibrium, this implies a mass within the half-light radius of $1.2_{-0.3}^{+0.4} \times 10^{7} \mathrm{M}_{\odot}$, and a massto-light ratio of $420 \mathrm{M}_{\odot} / L_{\odot}$, making Eri II a dark matter (DM) dominated satellite galaxy.

Not many satellite galaxies are known to host globular clusters (GCs). Only twelve out of $>76$ in the Local Group, and only three MW dSphs, Sagittarius, Fornax and Eri II host GCs (Grebel 2016). A small number of MW dSph satellites contain stellar substructures, e.g., Ursa Minor (Kleyna et al. 1998, 2004; Walker et al. 2006), Sextans; (Battaglia et al. 2011; Kim et al. 2019; Lora et al. 2019),

\footnotetext{
1 The papers by Simon et al. (2021) and Gallart et al. (2021) were submitted after this paper and their content was unknown to us in advance of publication.
} 


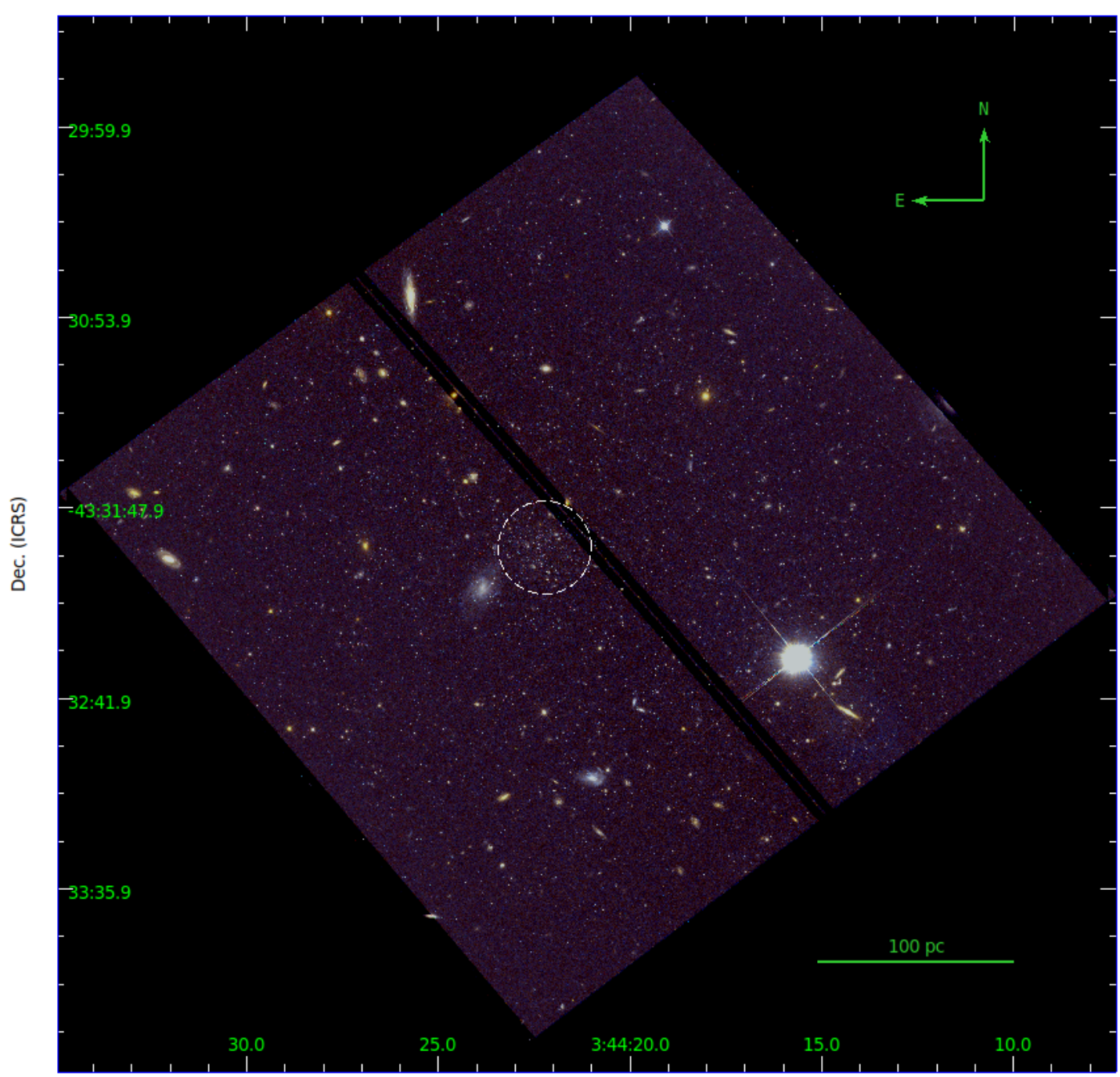

R.A. (ICRS)

Figure 1. HST/ACS image of Eri II in the WFC F814W band. The dashed circle encloses the structure identidied as a star cluster.

and Carina; (Lora et al. 2019). Eri II is thus one of the MW dSph galaxies that contains a star cluster.

From Dark Energy Survey (DES) public data, Koposov et al. (2015) identified a fuzzy object, a few parsecs in size, near the center of Eri II that they interpret as a GC. Crnojević et al. (2016) confirmed the presence of the partially resolved cluster. With $\mathrm{M}_{V}=-3.5$, the cluster accounts for 4\% of Eri II's total luminosity $\left(\mathrm{M}_{V}=-7.1\right)$. Eri II is then the least luminous galaxy known to host a star cluster.

From spectroscopic observations with the Multi Unit Spectroscopic Explorer (MUSE) on the Very Large Telescope (VLT), Zoutendijk et al. (2020) measured then mean line-of-sight velocity of 26 stars members of Eri II, seven of which were identified as possible cluster members. From the velocity and velocity dispersion distributions they confirm the existence of the cluster, conclude that its population is dynamically separated from the bulk of the stars of Eri II, and find no evidence of an excess of dark matter associated to the cluster.

Evidence for the presence of several stellar populations in Eri II has been reported in the literature. A major, old (> $10 \mathrm{Gyr})$ stellar population component was identified in Eri II by Crnojević et al. (2016), but it has been suggested that a younger population may also be present. Koposov et al. (2015) argue in favor of a second population as young as $250 \mathrm{Myr}$, but this claim was refused by Westmeier et al. (2015), who did not find any HI gas associated to Eri II. Crnojević et al. (2016) derived an HI mass limit of $M_{H I}<$ $2800 \mathrm{M}_{\odot}$, making Eri II a very gas-poor galaxy. Crnojević et al. (2016) also report a possible 3 Gyr old intermediate age population. Zoutendijk et al. (2020) assume an age of $8 \mathrm{Gyr}$ for both the bulk of the stars and the cluster in Eri II. However, they find one confirmed and two candidates carbon $(\mathrm{C})$ stars which indicate the presence of an intermediate-age population.

Analyzing deep HST phototometry Simon et al. (2021, S21 hereafter) determined the SFH of Eri II and the structural parameters of the star cluster. They show that at least $80 \%$ of the stars of Eri II formed before $z \sim 6$ (approximately 700 million years after the Big Bang). From a statistical analysis of the sub-haloes in the ELVIS simulation (Garrison-Kimmel et al. 2014) they conclude that probably Eri II has not yet passed through its closest approach to 
the MW, and therefore that reionization is then the most likely cause for the quenching of star formation in Eri II. However, Gallart et al. (2021, G21 hereafter) consider more plausible that the quenching of star formation in Eri II is due to stellar feedback by supernova events than to reionization. In favour of this idea they argue that the galaxy Leo T, with similar properties to Eri II, continued forming stars during a long period of time without being affected by reionization. Both S21 and G21 derive the SFH of Eri II comparing synthetic colour-magnitude diagrams (CMDs) with data using histograms. S21 use the statistical analysis of (Dolphin 2002) and G21 use the code TheStorm (Bernard et al. 2015, 2018).

The existence of stellar substructure in dSph galaxies is of major importance. Lora et al. $(2012,2013)$ studied the survival of old kinematic stellar substructures (e.g., star clusters) embedded in the DM halo of the Fornax and Sextans dSph galaxies using N-body simulations. The survival of stellar substructures within their DM halo suggests that the DM follows a core (flat) rather than a cuspy (NFW) central density profile (Navarro et al. 1997; Lora et al. 2012, 2013; Amorisco 2017; Contenta et al. 2018). The fact that Eri II is DM dominated and that it hosts a star cluster, makes it an ideal system to characterize its DM distribution.

In this paper we study in detail the stellar population(s) present in Eri II. The variety of age estimates for the stars in this galaxy motivates our study. To characterize the SFH of Eri II we avoid the isochrone fitting to the CMD and other data binning techniques, especially unappealing for sparse populations, that have been used frequently in the literature (Ramírez-Siordia et al. 2019). We opt for Bayesian inference, which allows us to estimate not only the physical parameters describing a stellar population but also their uncertainties. We apply the Bayesian hierarchical method developed by Alzate et al. (2021, A21 hereafter) to infer the age and metallicity distribution of resolved stellar populations, to characterize the physical properties of the bulk of the stars present today in Eri II. We attempt to date the star cluster in Eri II as a separate entity. Our method should detect intermediate age populations if present in Eri II.

The paper is organized as follows. In Section 2 we describe the HST data used in our analysis. In Section 3 we summarize the Bayesian hierarchical statistical method developed elsewhere by us to infer the star formation history (SFH) of resolved stellar populations. In Section 4 we infer the age and metallicity distribution of the stars observed in Eri II. In Appendix A we explore the ability of our method to detect or not more than one population of different age and metalliicty in a resolved galaxy and under what conditions. The conclusions are presented in Section 5.

\section{ERI II DATA}

In this work we use HST/ACS photometric data of Eri II in (a) the F606W and F814W bands published by S21, and $(b)$ the F475W and F814W bands derived by us from Hubble Legacy Archive images.

\subsection{F606W/F814W published photometry}

S21 include a photometric catalogue based on 20,680 s (F606W band) and 12,830 s (F814W band) exposures accumulated over 7 visits of the HST/ACS to Eri II as part of program GO-14234 (PI: J. D. Simon). These long exposures resulted in the most accurate photometry of Eri II available to date, with a signal-to-noise ratio of 10 for stars 1 mag below the main sequence turn-off (MSTO), almost four times the signal-to-noise ratio achieved by G21. The
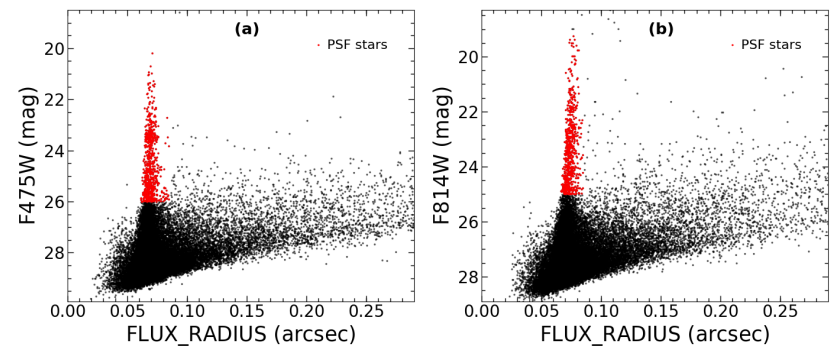

Figure 2. (MAG_AUTO, FLUX_RADIUS) plane for (a) F475W and (b) F814W. The black points represent the full initial catalogue and the red points the sources selected for the PSF model construction.

Table 1. Selection function for the PSF model.

\begin{tabular}{lcccc}
\hline Filter & $\begin{array}{c}\text { MAG_AUTO } \\
(\mathrm{mag})\end{array}$ & $\begin{array}{c}\text { FLUX_RADIUS }^{b} \\
(\operatorname{arcsec})\end{array}$ & $N_{S}^{c}$ & $N_{A}^{d}$ \\
\hline F606W & {$[1.2,1.7]$} & {$[20,26]$} & 677 & 498 \\
F814W & {$[1.2,1.7]$} & {$[19,25]$} & 654 & 431 \\
\hline
\end{tabular}

$a$ Kron-like (Kron 1980) elliptical aperture magnitude.

$b$ Estimated radius of the circle centered on the light barycenter enclosing half the total flux.

${ }^{c}$ Number of sources selected for the PSF model (red points in Fig. 2)

${ }^{d}$ Number of sources accepted by PSFEx.

S21 catalogue was derived using aperture and point spread function (PSF) photometry with the DAOPHOT-II package (Stetson 1987) in the STmag ${ }^{2}$ magnitude system. The $\mathrm{S} 21$ catalogue is shown as a CMD in Fig. 3b.

\subsection{F475W/F814W Hubble Legacy Archive images}

As part of program P.ID. 14224 (Cycle 23, P.I. C. Gallart, Gallart 2016, G16 hereafter), deep photometric data were obtained during 2 visits of the HST/ACS to Eri II, accumulating exposure times of $7644 \mathrm{~s}$ (F475W band) and $7900 \mathrm{~s} \mathrm{(F814W} \mathrm{band).} \mathrm{This} \mathrm{proposal} \mathrm{was}$ aimed to build a good quality CMD of Eri II reaching the MSTO with enough precision and accuracy to unveil the SFH of this galaxy. The exposures were distributed such that the telescope could collect the light of the brighter stars without losing the fainter stars, avoiding CCD saturation and allowing to build a complete CMD for both the bright and faint regimes. The long wavelength baseline provided by the filter pair (F475W, F814W) is ideal to study variations in age and metallicity in a resolved stellar population, requiring lower exposure times than other filter combinations to reach the necessary accuracy to separate the different stellar groups (see, e.g., Stetson 1994).

Before the photometric analysis, the two images available for each filter were combined using the routine IRAF/IMCOMBINE. PSF photometry was performed on each output image independently using the SExtractor ${ }^{3}$ and PSFEx ${ }^{4}$ software packages (Bertin \& Arnouts 1996; Bertin 2011). The latter is applied to build PSF models of the sources within a given image. SExtrator performance improves using PSF modeling. The source detection over the output

2 An object with constant flux $F_{\lambda}=3.63 \times, 10^{-9} \mathrm{erg} \mathrm{cm}^{-2} \mathrm{~s}^{-1} \AA^{-1}$ has magnitude STmag $=0$ in every filter. STmag $=-2.5 \log F_{\lambda}-21.1$.

3 http://www .astromatic.net/software/sextractor

4 https://www.astromatic.net/software/psfex 
a)

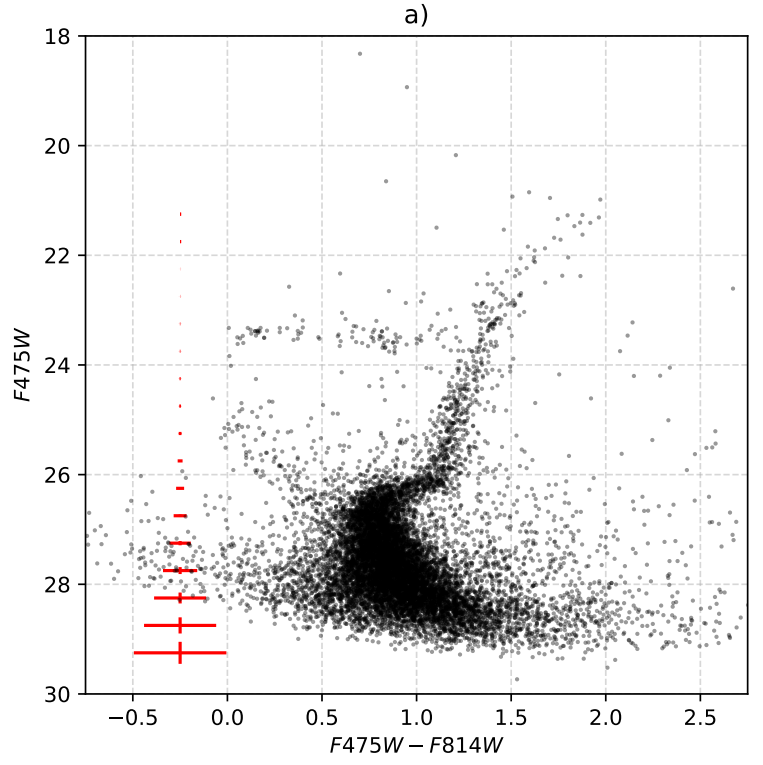

b)

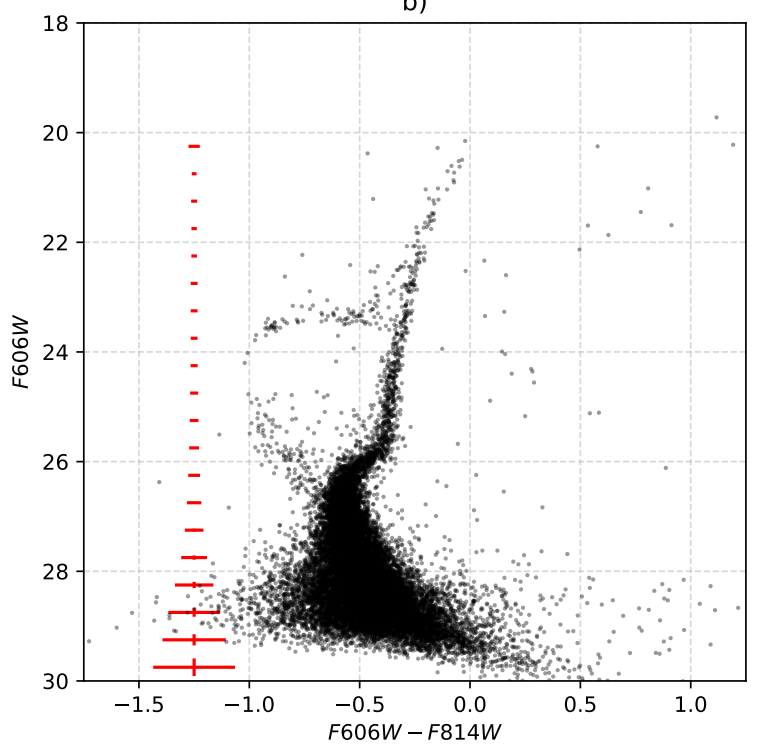

Figure 3. (a) F475W vs. F475W - F814W CMD (Vega mag) for 12,204 stars in the G16 data set. (b) F606W vs. F606W - F814W CMD (STmag) for 18,070 stars in the S21 data set.

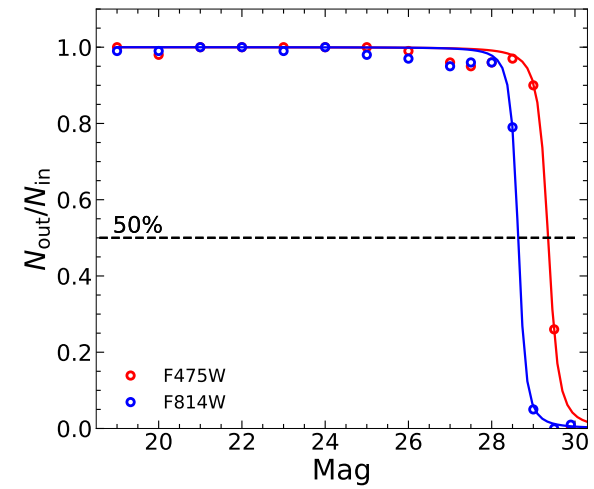

Figure 4. Completeness of our Eri II photometry. The vertical axis shows the ratio $N_{\text {out }} / N_{\text {in }}$ between the number of recovered and inserted artificial stars. The red and blue open dots show this ratio for stars detected according to our PSF model. The solid lines correspond to the fitted Pritchet function (Pritchet \& Harris 1990). The photometry is $90 \%$ complete at F475W $=28.5$ and $\mathrm{F} 814 \mathrm{~W}=29.0$.

images was done according to González-Lópezlira et al. (2017), summarized in the following steps: 1) an initial photometric catalogue is generated using SExtrator; 2) a set of sources are manually selected as shown in Figs. 2a and 2b; 3) PSFEx uses this selection to build the PSF model; and 4) a final source catalogue is produced running SExtractor a second time. Table 1 defines the selection function. Objects which satisfy these conditions are with high probability non-spurious sources.

Cross-matching the sky position of the sources and selecting those with FLAGS $\leq 4$ we obtain a sample of 12,159 stars with photometry in the two bands. SExtractor assigns FLAG $=0$ to well extracted sources and FLAGS $=1,2,4$ to sources affected by crowd-
Table 2. Sample completeness.

\begin{tabular}{|c|c|c|c|c|c|}
\hline \multirow{2}{*}{ Data set } & \multirow{2}{*}{ System } & \multicolumn{3}{|c|}{$90 \%$ completeness } & \multirow{2}{*}{$\mathrm{N}_{*}^{a}$} \\
\hline & & F475W & F606W & F814W & \\
\hline G16 & Vega & 29.0 & - & 28.5 & 10,670 \\
\hline $\mathrm{S} 21$ & STmag & - & 28.7 & 29.2 & 13,493 \\
\hline G21 & Vega & - & - & 28.5 & - \\
\hline
\end{tabular}

$a$ Number of stars defining $90 \%$ completeness.

ing issues ${ }^{5}$. We include sources flagged 1,2,4 to prioritize sample completeness. The resulting sample is shown as a CMD in Fig. 3a. The magnitudes from the G16 observations are in the Vega system.

\subsubsection{Sample completeness}

To determine the completeness of our photometry, we follow the add and recover artificial star method used by, e.g., González-Lópezlira et al. (2017); McLaughlin et al. (1994); Pritchet \& Harris (1990). We distribute artificially generated stars uniformly over the Eri II image, inserting 100 stars per magnitude interval in the range from 19 to 29 mag. We then repeat the extraction process described above. The number of artificial stars recovered up to a given magnitude defines the completeness limit for that magnitude. Fig. 4 shows our completeness function for the artificial stars using the Eri II PSF model. The sample is $\approx 90 \%$ complete at $\mathrm{F} 475 \mathrm{~W}=28.5$ and $\mathrm{F} 814 \mathrm{~W}=29.0$. G21 do not mention explicitly their completeness limits but their $\mathrm{CMD}$ extends to $\mathrm{F} 814 \mathrm{~W}=28.5$. Table 2 summarizes the completeness of the samples used in this paper.

\footnotetext{
5 https://sextractor.readthedocs.io/en/latest/index.html
} 


\section{METHODOLOGY}

The SFH of resolved stellar systems can be traced through isochrone fitting to their CMDs. Initially, a common method to fit theoretical isochrones to CMDs was based on subjective visual criteria. In the last twenty years objective quantitative methods based on $\chi^{2}$ minimization, maximum likelihood or Bayesian inference have become possible. The latter allow to estimate not only the physical parameters describing the stellar population but also their uncertainties. The case of galaxies with several bursts of star formation but poorly populated CMDs is particularly challenging since the number of free parameters is large enough to make binning methods unappealing (Ramírez-Siordia et al. 2019).

In this paper we use the Bayesian hierarchical method developed by A21 to infer the SFH of resolved stellar populations. The $\mathrm{SFH}$ is expressed as a linear combination of $N_{i}$ stellar populations, each one described by an isochrone of known age and metallicity (Dolphin 1997; Small et al. 2013). From the posterior probability distribution function (PDF) we obtain the relative contribution $a_{i}$ of the $i^{t h}$ isochrone with its corresponding confidence interval. Since data binning is not required, this method is well suited to study populations with small numbers of stars, down to a few hundreds.

In a Bayesian hierarchical scheme we have two kind of parameters: population parameters, like $a_{i}$ (proportional to the star formation rate, SFR) and $\phi$ (the initial mass function, IMF), and individual parameters, like $M_{j}^{k}$, the absolute magnitude of the $j^{t h}$ star in the $k$ band according to our isochrones. $k=1,2$ correspond to the F475W and F814W bands, respectively, in the case of the G16 data set, or to the F606W and F814W bands, respectively, for the S21 sample. The population parameters determine the PDF of the individual parameters. The Galactocentric distance $r_{E r i}$ and the extinction $A_{k}$ are taken as fixed quantities, as well as the set of isochrones which determine $M_{i}^{k}$. The locus described by each isochrone in the CMD is parametrized as a function of the stellar mass $m . M_{i}^{k}$ is then a unique function of $m$ for the $i^{t h}$ isochrone in the $k^{t h}$ band. We model the absolute magnitude of a star of mass $m$ as a random variable of a convoluted isochrone such that $M_{j}^{k} \sim \mathcal{N}\left(\mathcal{M}_{i}^{k}(m), \sigma_{i}^{k}\right)$. For simplicity, the dispersion $\sigma_{i}^{k}$ is taken as a constant, i.e., the isochrone is dispersed uniformly near each value of $m$. The posterior PDF of the population parameters given the data $F_{j}^{k}$ with error $e_{k}^{k}$ follows then from Bayes theorem (A21)

$\mathrm{P}\left(\boldsymbol{a} \mid F_{j}^{k}\right) \propto \mathrm{P}(\boldsymbol{a}) \prod_{j=1}^{N_{D}} \int \frac{S\left(F_{j}^{k}\right) \mathrm{P}\left(F_{j}^{k} \mid F_{\text {true }}^{k}\right) \mathrm{P}\left(M_{j}^{k} \mid \boldsymbol{a}\right)}{\ell(\boldsymbol{a}, S)} d M_{j}^{k}$,

where $S\left(F_{j}^{k}\right)$ is the completeness function, a constant for a complete sample, and $\mathrm{P}\left(F_{j}^{k} \mid F_{\text {true }}^{k}\right)$ is the likelihood function, taken as the normal distribution

$\mathcal{N}\left(F_{j}^{k} \mid F_{\text {true }, j}^{k}, e_{j}^{k}\right) \propto \exp \left(\frac{F_{j}^{k}-F_{\text {true }}}{e_{j}^{k}}\right)^{2}$.

$F_{\text {true }}^{k}=M_{j}^{k}+\mu$ is the true apparent magnitude, an ideal measurement with no uncertainty, which is always unknown but it is needed for the statistical model structure (Luri et al. 2018). $\mu$ is the distance modulus.

In Eq. (1) the denominator $\ell(\boldsymbol{a}, S)$ is a normalization constant, and the hyper-prior $\mathrm{P}(\boldsymbol{a})$, where $\boldsymbol{a}=\left\{a_{i=1, \ldots, N_{I}}\right\}, a_{i}>0$ and $\sum_{i} a_{i}=1$, is the symmetric Dirichlet distribution (Walmswell et al.
2013). The prior $\mathrm{P}\left(M_{j}^{k} \mid \boldsymbol{a}\right)$ is defined in $\mathrm{A} 21$ as

$P\left(M_{j}^{k} \mid \mathbf{a}\right) \propto \sum_{i=1}^{N_{i s o}} a_{i} \int_{m_{l, i}}^{m_{u, i}} \phi(m) \prod_{k=1}^{3} \mathcal{N}\left(M_{j}^{k} \mid \mathcal{M}_{i}^{k}(m), \sigma_{i}^{k}\right) d m$.

In Eq. (3) the IMF $\phi(m)$, the lower and upper mass limits $\left(m_{l}, m_{u}\right)$, and the isochrones enter as fixed quantities. See A21 for details.

For a complete sample the distribution of the number of stars along the MS is determined by the IMF. If the sample is complete to a limiting magnitude, it is necessary to renormalize the statistical model to compensate for the missing stars. If we truncate the sample to this limiting magnitude, the integral in Eq. (1) must be truncated to this limit. In this way we ensure that the posterior PDF is correctly normalized according to the number of stars in the IMF.

\subsection{Stellar tracks and isochrones}

We use two independent sets of isochrones for our Bayesian inference.

\subsubsection{PARSEC isochrones}

The PARSEC isochrones ${ }^{6}$ (Bressan et al. 2012) are available for different stellar mass ranges depending on the stellar metallicity $Z$. For $0.0001 \leq Z \leq 0.02$ the isochrones extend from 0.1 to $350 \mathrm{M}_{\odot}$; for $0.03 \leq Z \leq 0.04$ from 0.1 to $150 \mathrm{M}_{\odot}$; and for $Z=0.06$ from 0.1 to $20 \mathrm{M}_{\odot}$. We use their $[\alpha / \mathrm{Fe}]=0$ (scaled-solar) isochrones, which include overshooting, atomic diffusion and the parameter $\eta$ describing the mass loss rate in the red giant branch (RGB) is set to $\eta=0.2$.

\subsubsection{BaSTI isochrones}

The BaSTI isochrones ${ }^{7}$ (Hidalgo et al. 2018) are available for $0.00001 \leq Z \leq 0.03905$ covering the mass range from 0.1 to $15 \mathrm{M}_{\odot}$. We use their scaled-solar isochrones, including overshooting, atomic diffusion, and $\eta=0.3$.

\subsection{Isochrone grids}

From the BaSTI and PARSEC isochrone libraries, we assemble the two grids of isochrones listed in Table 3 that will be used below to infer the age-metallicity distribution of Eri II. We remark here that the $Z=0.00001$ and 0.00005 isochrones, available in the BaSTI data set, are not available for the PARSEC set.

\section{AGE-METALLICITY DISTRIBUTION OF ERI II}

To infer the age-metallicity distribution (AMD) from the CMD we proceed as follows. The isochrones listed in Table 3 define the loci occupied in the CMD by stars of the corresponding age and metallicity. The isochrones are parametrized by the stellar mass and then re-sampled such that the difference in magnitude between consecutive points along the isochrone is $\approx 0.005 \mathrm{mag}$. To build the posterior PDF we sample the $a_{i}$ parameter space using a $10^{5}$

\footnotetext{
6 http://stev.oapd.inaf.it/cgi-bin/cmd

7 http://basti-iac.oa-abruzzo.inaf.it
} 
Table 3. Isochrone Grids

\begin{tabular}{|c|c|c|c|c|c|c|c|c|c|c|}
\hline Grid & Isochrones & $\begin{array}{l}\text { Age } \\
(\mathrm{Gyr})\end{array}$ & $\begin{array}{l}\text { Step } \\
(\mathrm{Gyr})\end{array}$ & 0.00001 & 0.00005 & $\begin{array}{r}Z \\
0.0001\end{array}$ & 0.0002 & 0.0005 & 0.001 & $\mathrm{~N}_{\text {iso }}$ \\
\hline A & BaSTI & {$[1,14]$} & 0.5 & $\checkmark$ & $\checkmark$ & $\checkmark$ & $\checkmark$ & $\checkmark$ & & 135 \\
\hline B & PARSEC & {$[1,14]$} & 0.5 & & & $\checkmark$ & $\checkmark$ & $\checkmark$ & $\checkmark$ & 108 \\
\hline
\end{tabular}

a)
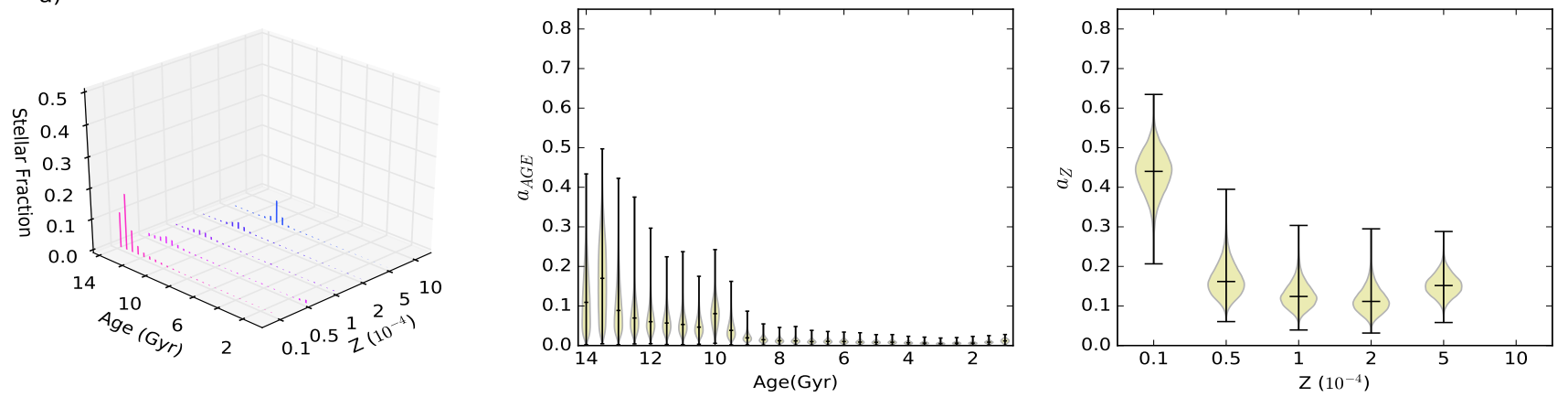

b)
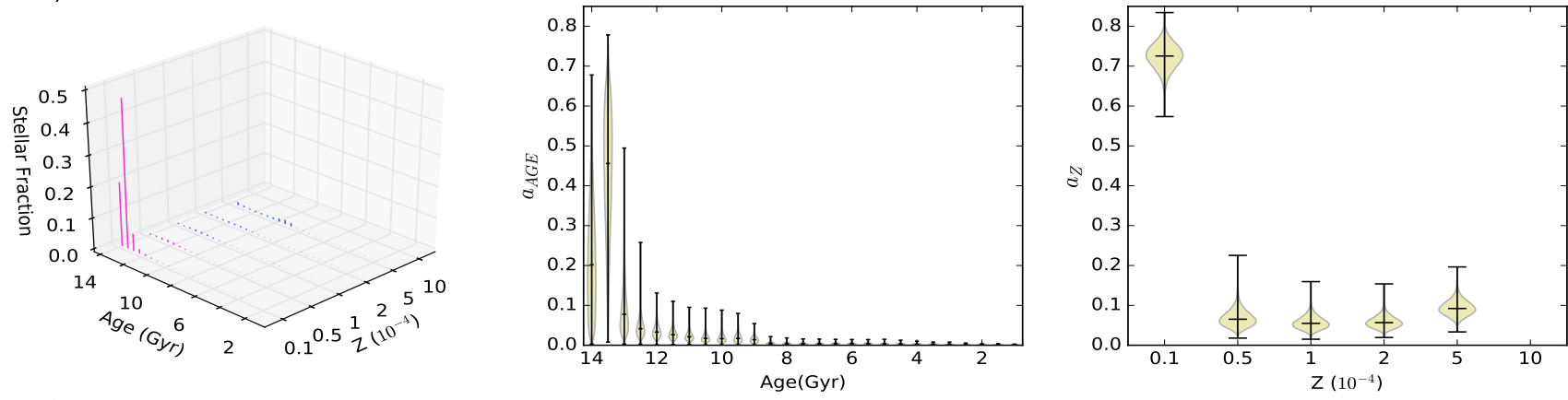

c)
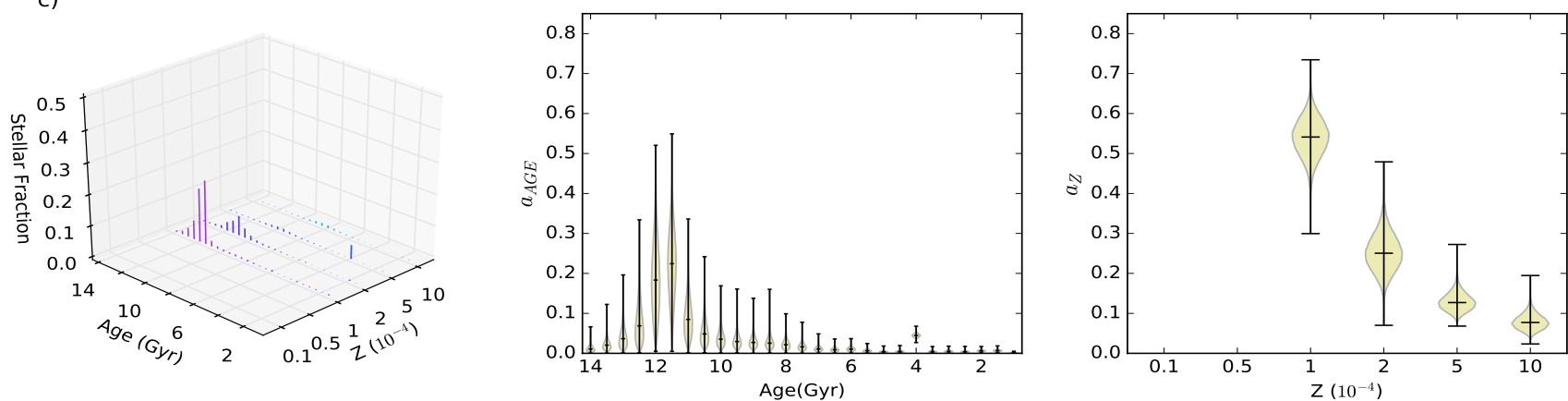

d)
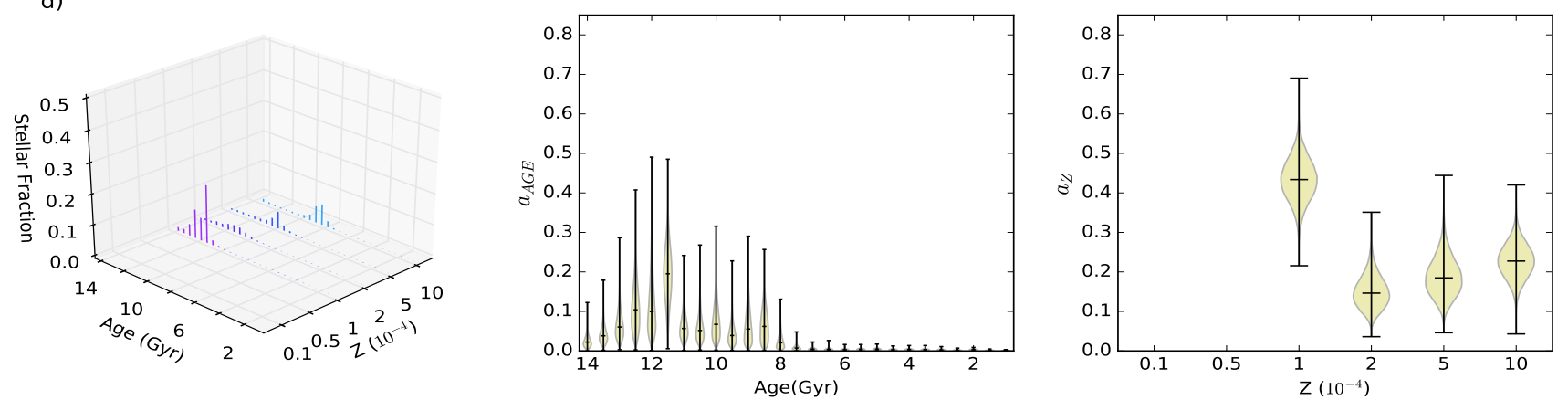

Figure 5. AMD of Eri II inferred from the G16 and S21 data sets using isochrone grids A and B (Table 3). Our analysis is limited to the $90 \%$ sample completeness regime. (a) G16 data set and isochrone grid A and $\sigma_{i}^{k}=0.05$ mag. (b) $\mathrm{S} 21$ data set and isochrone grid A and $\sigma_{i}^{k}=0.03$ mag. (c) G16 data set and isochrone grid B and $\sigma_{i}^{k}=0.05 \mathrm{mag}$. (d) $\mathrm{S} 21$ data set and isochrone grid B and $\sigma_{i}^{k}=0.03 \mathrm{mag}$. 
a)

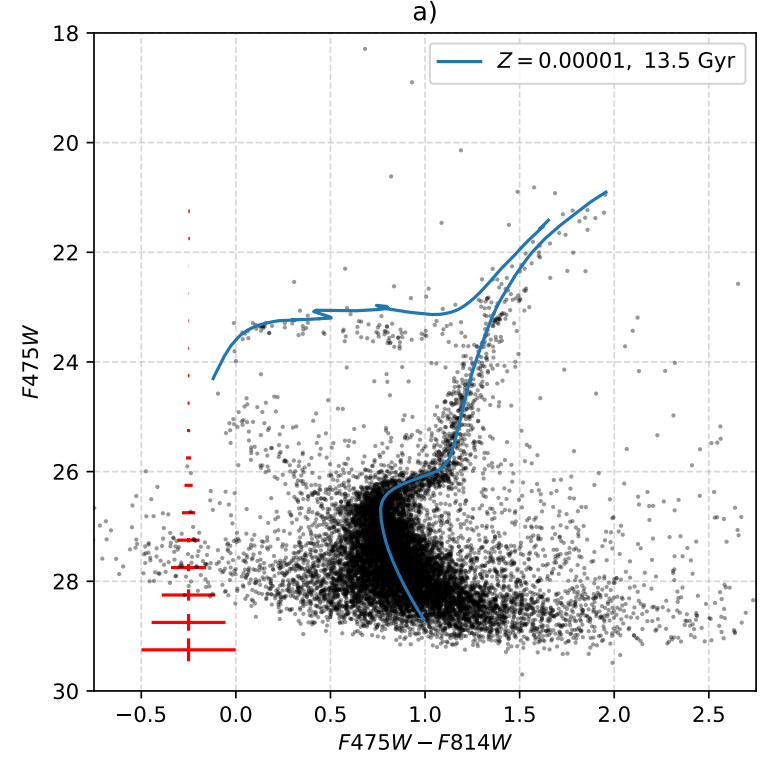

c)

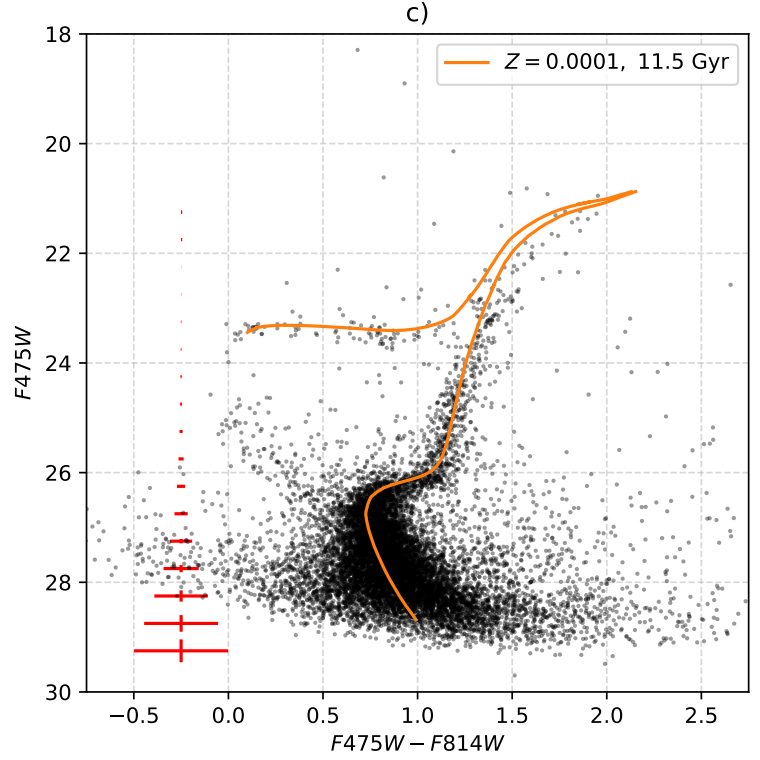

b)

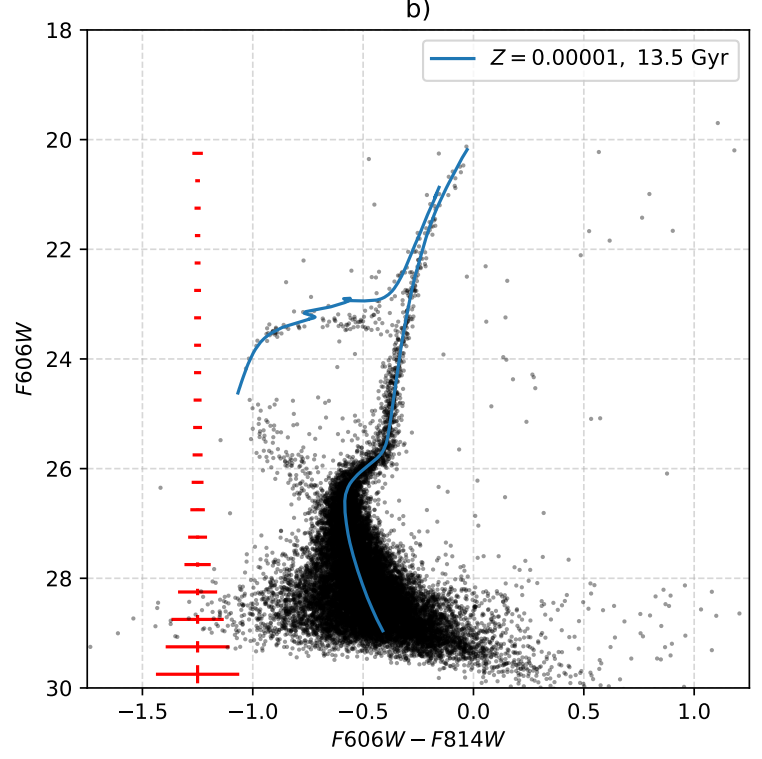

d)

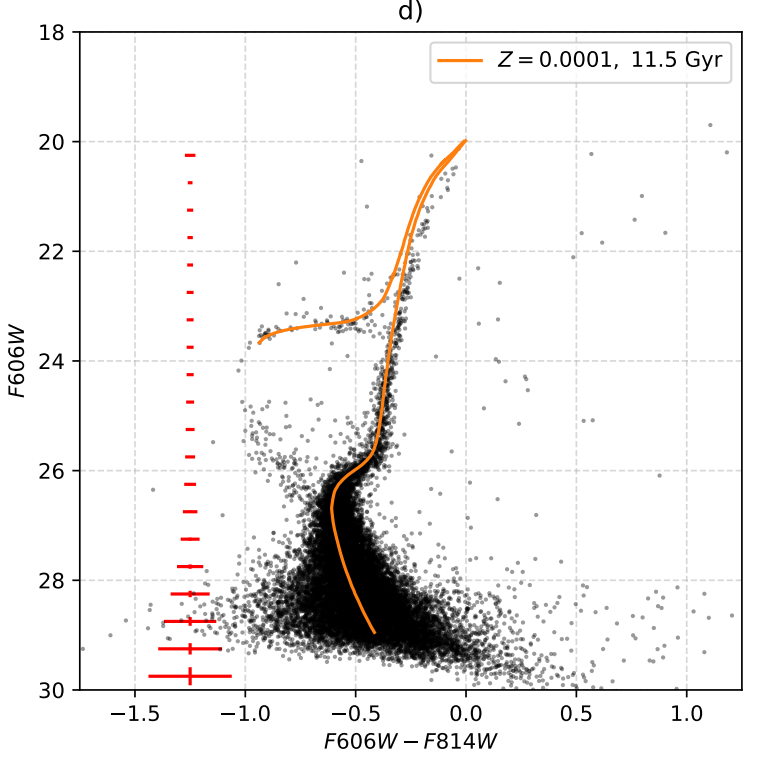

Figure 6. $(a, c)$ F475W vs. F475W - F814W CMD (Vega mag) for 12,204 stars in the G16 data set. (b,d) F606W vs. F606W - F814W CMD (STmag) for 18,070 stars in the S21 data set. The $13.5 \mathrm{Gyr}, Z=0.00001 \mathrm{BaSTI}$ isochrone, corresponding to the maximum value of $a_{A G E}$ in Figs. 5a,b, is shown as a blue line in the upper row panels. The $11.5 \mathrm{Gyr}, Z=0.0001$ PARSEC isochrone, corresponding to the maximum value of $a_{A G E}$ in Figs. 5c,d, is shown as an orange line in the bottom row panels.

Table 4. Information Criteria.

\begin{tabular}{cccccc}
\hline Figure & Data & Grid & $\begin{array}{c}\text { Maximum } \\
\text { Likelihood }\end{array}$ & BIC & AIC \\
\hline $5 a$ & G16 & A & -12669 & 26592 & 25608 \\
$5 b$ & S21 & A & -6537 & 14361 & 13345 \\
& & & & & \\
$5 c$ & G16 & B & -14943 & 30890 & 30102 \\
$5 d$ & S21 & B & -6970 & 14970 & 14157 \\
\hline
\end{tabular}

step Markov chain Monte Carlo (MCMC) process. We use the Stan MCMC platform ${ }^{8}$ due to its recognized reliability and acceptance of hard constraints, e.g., $a_{i} \geq 0$ and $\sum a_{i}=1$, as required in our case. From this posterior we infer the distribution of values of $a_{i}$ and its confidence interval. We assume the Kroupa (2001) IMF.

In this paper we use $\mu=22.8$ for the distance modulus to Eri II, as reported by Crnojević et al. (2016). From the NED ${ }^{9}$ database

\footnotetext{
8 https://mc-stan.org

9 https://ned.ipac. caltech.edu
} 

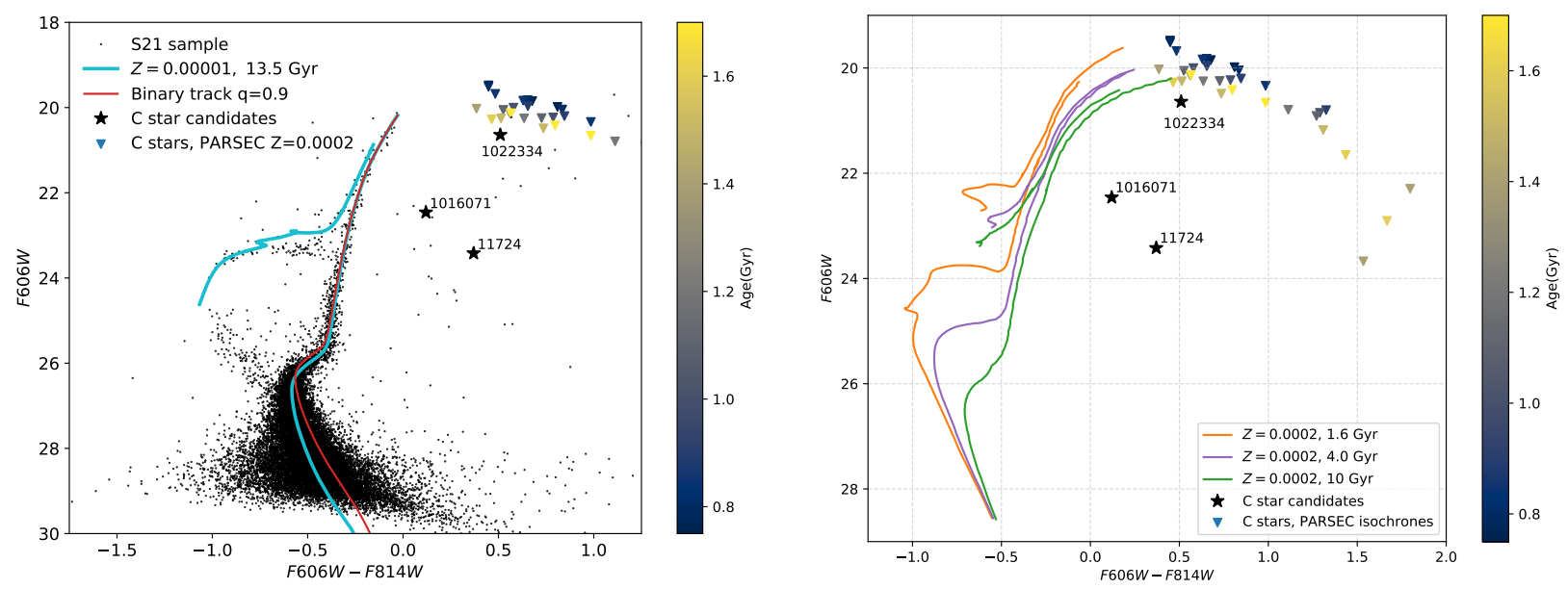

Figure 7. F606W - F814W vs. F606W CMD in STmag units indicating the position of the Zoutendijk et al. (2020) C star candidates, and the C stars for the $Z=0.0002$ PARSEC isochrones. No photometric errors are provided by Zoutendijk et al. (2020). The cyan line in the left-hand-side panel is the 13.5 Gyr, $Z=0.00001$ single star BaSTI isochrone, corresponding to the maximum value of $a_{A G E}$ in Figs. $5 \mathrm{~b}$. The red line in this panel corresponds to the expected position of this isochrone in the CMD when unresolved binary stars of mass ratio $q=m_{2} / m_{1}=0.9$ are taken into account. Single star PARSEC isochrones are shown in the right-hand-side panel.

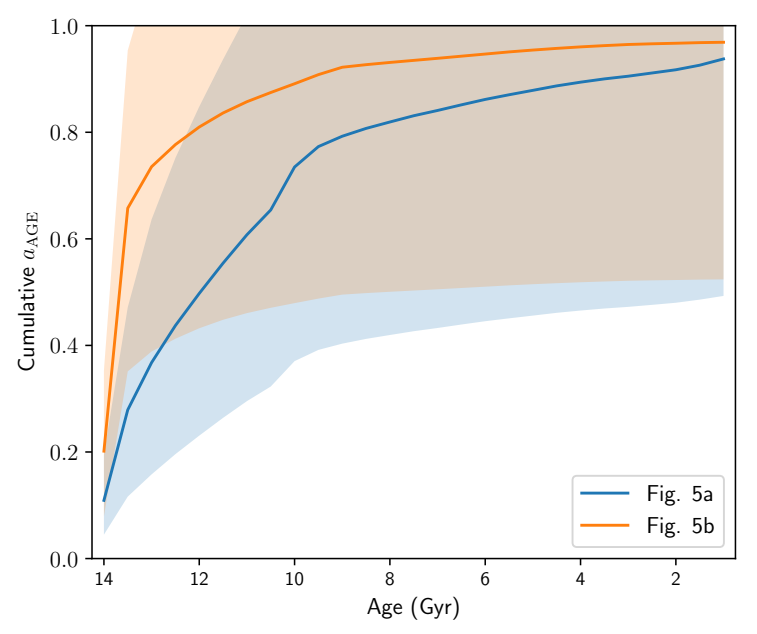

Figure 8. Normalized cumulative number of stars vs. age corresponding to the AMDs in Fig. 5a (blue line, G16, isochrone Grid A) and Fig. 5b (orange line, S21, isochrone grid A). The shaded areas indicate the percentiles 10 and 90 of the corresponding distribution.

we obtain the extinction in the direction of Eri II in the 3 bands in use $\left(A_{475}, A_{606}, A_{814}\right)=(0.033,0.025,0.015) \mathrm{mag}$.

The AMDs inferred from the G16 and S21 Eri II samples (Section 2 and Fig. 3) using isochrone grids A and B (Table 3) are displayed in Fig. 5. The height of the bars in the 3D plots (left panels) of Fig. 5 is the median of the distribution of $a_{i}$ for the corresponding isochrone. The violin plots summarize the marginalized posterior PDF for age (center panels) and $Z$ (right panels). The horizontal lines in each violin represent from bottom to top the 0,50 , and 100 percentiles of the distribution, respectively. The age-metallicity degeneracy (Worthey 1994) is clearly noticeable in these distributions: the higher $Z$ PARSEC isochrones (Grid B) predict younger ages for the Eri II stellar population than the lower $Z$ BaSTI isochrones
(Grid A). We remind the reader that the $Z=0.00001$ and 0.00005 isochrones are not available for the PARSEC set in Grid B.

The agreement between the AMD's in Figs. $5 a, b$ and Figs. $5 c, d$ is quite remarkable given the different photometries and isochrones in use. In Table 4 we list the values for the Bayesian information criterion (BIC) and the Akaike information criterion (AIC) for the four inferences in Fig. 5. These criteria ${ }^{10}$ are partially based on the likelihood function and serve for model selection among a finite set of models (Gelman et al. 2013). Though BIC is always higher than AIC, the lower the value of these two measures, the better the model. Thus, the model in $5 b$ describes the data better than the model in $5 a$. Similarly, the model in $5 d$ describes the data better than the model in $5 c$. The similarities between the inferred AMD's for these two pairs of data and models support that the stellar evolution models play an important role. The solutions consistently compensate the lack of the most metal-poor models with younger ages.

In Fig. 6 we show the isochrones corresponding to the maximum $a_{A G E}$ in the AMDs of Fig. 5. Comparing Fig. 6b,d, it is apparent that the RGB is reproduced better by the BaSTI than by the PARSEC isochrones, even though the opposite may be true for the horizontal branch (HB) stars. To a lesser degree, the same behaviour is observed when comparing Fig. 6a,c. For this reason we consider the AMD in Fig. $5 b$ to be the most realistic approximation to the true distribution. From Fig. 8 we see that according to this $\mathrm{AMD}, \approx 80 \%$ of the stars in Eri II are older than 13 Gyr. In contrast, the solution for the G16 data set (Fig. 5a) implies that $\approx 60 \%$ of the stars in Eri II are younger than 13 Gyr. This discrepancy is likely due to the lower quality of the G16 (shorter exposures, lower signal-to-noise ratio, less deep images) compared to the S21 data set, and possibly indicates that we underestimated the photometric errors for the G16 sample (see Section 2).

$10 B I C=\ln (n) k-2 \ln (\mathcal{L})$, where $\mathcal{L}$ is the maximum likelihood value, $n$ the number of data points, and $k$ the number of estimated free parameters. $A I C=2 k-2 \ln (\mathcal{L})$ 


\subsection{Carbon stars in Eri II}

Zoutendijk et al. (2020) identified three possible C stars in the field of Eri II. One of them (ID1022334) was confirmed to be a C star and to belong to the bulk population of Eri II, the other two (ID11724, ID1016071) remain as candidates. The presence of C stars supports the existence of an intermediate age population in Eri II. According to the $Z=0.0002$ PARSEC evolutionary tracks ${ }^{11}$, stars of MS mass in the range 2.2 to $1.55 \mathrm{M}_{\odot}$ become $\mathrm{C}$ stars at age from 0.75 to $1.7 \mathrm{Gyr}$ old, respectively. The age of $\mathrm{C}$ stars increases slowly with metallicity, reaching $\approx 2 \mathrm{Gyr}$ near $Z_{\odot}$. Since we do not detect any residual star formation extending to $\approx 2 \mathrm{Gyr}$, it is likely that the $\mathrm{C}$ stars evolve from lower mass progenitors that increased their mass through stellar fusions (blue-stragglers, seen in significant numbers in the CMDs). This mechanism requires the star density in Eri II to be high enough for stellar collisions to be important.

In Fig. 7 we show the position of the Zoutendijk et al. (2020) C star candidates in the CMD together with the $\mathrm{C}$ stars expected from the $Z=0.0002$ PARSEC isochrones for $0.75 \leq$ age $\leq 1.7$ Gyr. Only the confirmed candidate ID1022334 has the luminosity and colour corresponding to $\mathrm{C}$ stars. The $\mathrm{C}$ star nature of the other two candidates seems doubtful. Part of the difference between models and observations in Fig. 7 may result from inaccuracies in the Aringer et al. (2009) C star spectral models used to compute the expected C star colors. In this case we used the $Z=0.1 \times Z_{\odot}$ models, which is the minimum metallicity for which models are available. Taken at face value, the observed $(\mathrm{F} 606 \mathrm{~W}, \mathrm{~F} 814 \mathrm{~W})=(20.64,20.13) \mathrm{STmag}$ place star ID1022334 at 500 to $400 \mathrm{kpc}$ from us, $\mu=(23.5,23.05)$ mag, respectively, while the reported $\mu$ to Eri II is 22.83 mag (Crnojević et al. 2016).

\subsection{Dating the star cluster in Eri II}

We made several attempts to date the star cluster in Eri II as a separate entity, with no success. We selected all the stars inside the dashed white circle in Fig. 1. The number of cluster stars is too low $(\approx 300)$ for our Bayesian hierarchical model to work properly. Our grids contain $\sim 100$ isochrones, so in this case we have $\approx 3$ stars per isochrone, which prevents a proper statistical treatment of the cluster population. In Appendix A we use simulated stellar populations to show that this is in fact the case.

\section{CONCLUSIONS}

We have performed a detailed stellar population analysis of Eridanus II using our Bayesian hierarchical model (Alzate et al. 2021) to infer with an acceptable level of statistical significance relevant information about its SFH.

We find convincing evidence that the bulk of the stars in Eri II are very old, with an age of $13.5_{-1}^{+0.5} \mathrm{Gyr}$ and quite metal poor, with $Z=0.00001$ (see the AMD in Fig. 5b). In agreement with S21, we found that the $70 \%$ of the stars were formed 700 Myr after Big Bang (Fig. 8). This result is consistent with the width at half maximum ( $~ 500 \mathrm{Myr}$ ) of the derived star formation rate profile of G21.

We did not succeed in determining the age of the star cluster as an independent entity due in part to the small number of visible stars directly associated with the cluster. Nor we find any evidence of the presence of an intermediate age population. Cluster stars are

11 We do not have available this information for the BaSTI tracks. expected to be included in the stellar population that we analyzed with no indication of bimodal distributions in age or in metallicity.

The survival of the star cluster for over $13 \mathrm{Gyr}$ inside the DM halo of Eri II favours the model of a flat (core) rather than a cuspy (NFW) central density profile (Lora et al. 2012, 2013, 2020 in preparation).

The lack of recent star formation implies that mass pumping of lower mass MS stars through blue-straggler fusions is responsible of forming the massive progenitors of the $\mathrm{C}$ stars seen today in Eri II.

From simulated stellar populations we conclude that $(a)$ we can recover correctly the age of stellar populations of the same metallicity born in different star formation episodes separated in time by more than the time resolution of the isochrone grid in use; (b) nearly coeval populations of different metallicity can be characterized as long as the number of stars in the less massive population is significant; and (c) the size of the photometric errors included in the statistical model must resemble the true errors.

\section{ACKNOWLEDGEMENTS}

We thank the anonymous referee for the careful reading of our manuscript and for useful suggestions that improved the quality and scope of this paper. We thank very specially Denija Crnojevich who kindly guided us through the Eridanus II data. GB and JAA acknowledge financial support from the National Autonomous University of México (UNAM) through grant DGAPA/PAPIIT IG100319 and from CONACyT through grant CB2015-252364. VL gratefully acknowledges support from the CONACyT Research Fellowship program. BCS acknowledges financial support through PAPIIT project IA103520 from DGAPA-UNAM. The research in this paper is part of the $\mathrm{PhD}$ thesis of J. A. Alzate in the Universidad Nacional Autónoma de México (UNAM) graduate program in astrophysics. He thanks the support from the Instituto de Radioastronomía and Astrofísica, its staff, and the Consejo Nacional de Ciencia y Tecnología (CONACyT) for the scholarship granted.

This work is based on observations made with the NASA/ESA Hubble Space Telescope, and obtained from the Hubble Legacy Archive, which is a collaboration between the Space Telescope Science Institute (STScI/NASA), the Space Telescope European Coordinating Facility (ST-ECF/ESAC/ESA) and the Canadian Astronomy Data Centre (CADC/NRC/CSA).

\section{DATA AVAILABILITY}

The Gallart (2016) Eridanus II data underlying this article are available in the Hubble Legacy Archive, Proposal ID 14224, 2016, (https://hla.stsci.edu/hlaview.html). The Simon et al. (2021) Eridanus II photometry used in this article is available in the astro-ph, arXiv:2012.00043, 2020 (https://arxiv.org/abs/2012.00043). We make use of the PARSEC isochrones (Bressan et al. 2012, http:// stev.oapd.inaf.it/cgi-bin/cmd), the BaSTI isochrones (Hidalgo et al. 2018, http://basti-iac.oa-abruzzo.inaf.it), the Stan MCMC platform (https://mc-stan.org), the NED database (https://ned.ipac.caltech.edu), and the Bertin \& Arnouts (1996) SExtractor (http://www.astromatic.net/ software/sextractor) and Bertin (2011) PSFEx (https:// www.astromatic.net/software/psfex) software packages. 


\section{REFERENCES}

Alzate J. A., Bruzual G., Díaz-González D. J., 2021, MNRAS, 501, 302

Amorisco N. C., 2017, ApJ, 844, 64

Aringer B., Girardi L., Nowotny W., Marigo P., Lederer M. T., 2009, A\&A, 503, 913

Battaglia G., Tolstoy E., Helmi A., Irwin M., Parisi P., Hill V., Jablonka P., 2011, MNRAS, 411, 1013

Bechtol K., et al., 2015, ApJ, 807, 50

Bernard E. J., Ferguson A. M. N., Chapman S. C., Ibata R. A., Irwin M. J., Lewis G. F., McConnachie A. W., 2015, MNRAS, 453, L113

Bernard E. J., Schultheis M., Di Matteo P., Hill V., Haywood M., Calamida A., 2018, MNRAS, 477, 3507

Bertin E., 2011, in Evans I. N., Accomazzi A., Mink D. J., Rots A. H., eds, Astronomical Society of the Pacific Conference Series Vol. 442, Astronomical Data Analysis Software and Systems XX. p. 435

Bertin E., Arnouts S., 1996, A\&AS, 117, 393

Bressan A., Marigo P., Girardi L., Salasnich B., Dal Cero C., Rubele S., Nanni A., 2012, MNRAS, 427, 127

Contenta F., et al., 2018, MNRAS, 476, 3124

Crnojević D., Sand D. J., Zaritsky D., Spekkens K., Willman B., Hargis J. R., 2016, ApJ, 824, L14

Dolphin A., 1997, New Astron., 2, 397

Dolphin A. E., 2002, MNRAS, 332, 91

Gallart C., 2016, Hubble Legacy Archive, Eridanus II, Proposal ID 14224, https://hla.stsci.edu/hlaview.html

Gallart C., et al., 2021, ApJ, 909, 192

Garrison-Kimmel S., Boylan-Kolchin M., Bullock J. S., Lee K., 2014, MNRAS, 438, 2578

Gelman A., Carlin J., Stern H., Dunson D., Vehtari A., Rubin D., 2013, Bayesian Data Analysis, Third Edition. Chapman \& Hall/CRC Texts in Statistical Science, Taylor \& Francis, https ://books .google.com. $\mathrm{mx} /$ books? id=ZXL6AQAAQBAJ

González-Lópezlira R. A., et al., 2017, ApJ, 835, 184

Grebel E. K., 2016, in Meiron Y., Li S., Liu F. K., Spurzem R., eds, IAU Symposium Vol. 312, Star Clusters and Black Holes in Galaxies across Cosmic Time. pp 157-170

Grebel E. K., Gallagher John S. I., 2004, ApJ, 610, L89

Hidalgo S. L., et al., 2018, ApJ, 856, 125

Kim H.-S., Han S.-I., Joo S.-J., Jeong H., Yoon S.-J., 2019, ApJ, 870, L8

Kleyna J. T., Geller M. J., Kenyon S. J., Kurtz M. J., Thorstensen J. R., 1998, AJ, 115, 2359

Kleyna J. T., Wilkinson M. I., Evans N. W., Gilmore G., 2004, MNRAS, 354, L66

Koposov S. E., Belokurov V., Torrealba G., Evans N. W., 2015, ApJ, 805, 130

Kroupa P., 2001, MNRAS, 322, 231

Li T. S., et al., 2017, ApJ, 838, 8

Lora V., Just A., Sánchez-Salcedo F. J., Grebel E. K., 2012, ApJ, 757, 87

Lora V., Grebel E. K., Sánchez-Salcedo F. J., Just A., 2013, ApJ, 777, 65

Lora V., Grebel E. K., Schmeja S., Koch A., 2019, ApJ, 878, 152

Luri X., et al., 2018, A\&A, 616, A9

McLaughlin D. E., Harris W. E., Hanes D. A., 1994, ApJ, 422, 486

Muñoz R. R., Côté P., Santana F. A., Geha M., Simon J. D., Oyarzún G. A., Stetson P. B., Djorgovski S. G., 2018, ApJ, 860, 66

Navarro J. F., Frenk C. S., White S. D. M., 1997, ApJ, 490, 493

Pritchet C. J., Harris W. E., 1990, ApJ, 355, 410

Ramírez-Siordia V. H., Bruzual G., Cervantes Sodi B., Bitsakis T., 2019, MNRAS, 486, 5567

Simon J. D., et al., 2021, ApJ, 908, 18

Small E. E., Bersier D., Salaris M., 2013, MNRAS, 428, 763

Stetson P. B., 1987, PASP, 99, 191

Stetson P. B., 1994, PASP, 106, 250

Walker M. G., Mateo M., Olszewski E. W., Pal J. K., Sen B., Woodroofe M., 2006, ApJ, 642, L41

Walmswell J. J., Eldridge J. J., Brewer B. J., Tout C. A., 2013, MNRAS, 435,2171

Westmeier T., Staveley-Smith L., Calabretta M., Jurek R., Koribalski B. S.,
Meyer M., Popping A., Wong O. I., 2015, MNRAS, 453, 338

Worthey G., 1994, ApJS, 95, 107

Zoutendijk S. L., et al., 2020, A\&A, 635, A107

\section{APPENDIX A: AMD RECOVERY BY BAYESIAN INFERENCE}

\section{A1 Simulated CMDs}

In this section we explore the minimal separation in age, metallicity and number of stars, (i.e., resolution) that we can detect with our Bayesian inference scheme using a set of simulated stellar populations. In these simulations we assume two star formation episodes, listed in Table A1: (a) a long constant star formation rate burst starting $12.25 \mathrm{Gyr}$ ago and ending $11.75 \mathrm{Gyr}$ ago, with a burst duration of $0.5 \mathrm{Gyr}$; and $(b)$ an instantaneous burst happening $0.25 \mathrm{Gyr}$ or 0.75 Gyr after the end of the previous event.

For simplicity we use the simulated population option of the PARSEC isochrone web interface ${ }^{12}$ to generate the simulated populations. Stars in event $(a)$ belong to three isochrones, dated 11.75, 12 and 12.25 Gyr. Stars in event $(b)$ belong to a single isochrone, dated either 11 or 11.5 Gyr. The PARSEC simulations assume the Kroupa (2001) IMF. The number of stars formed in event $(a)$ is $\approx 2.5$ times the number of stars formed in event $(b)$ for simulations 1,2 and 3 , and $\approx 16$ times for simulations 4 and 5 (see Table A1).

We obtain the apparent magnitudes $\left(\mathfrak{m}_{F 475 W}, \mathfrak{m}_{F 814 w}\right)$ of each star adding the distance modulus of Eri II, $\mu=22.8$ mag (Crnojević et al. 2016) to the absolute magnitudes $\left(M_{\mathrm{F} 475 \mathrm{~W}}, M_{\mathrm{F} 814 \mathrm{~W}}\right)$ output by the simulations in the Vega magnitude system. We add the photometric error to the magnitudes of each star by random

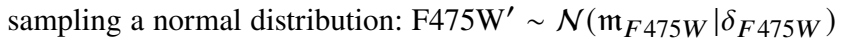
and $\mathrm{F} 814 \mathrm{~W}^{\prime} \sim \mathcal{N}\left(\mathrm{m}_{F 814 W} \mid \delta_{F 814 W}\right)$, where the prime symbols refer to the simulated apparent magnitudes. The magnitude errors $\delta_{\mathrm{F} 475 \mathrm{~W}}$ and $\delta_{\mathrm{F} 814 \mathrm{~W}}$ were derived from the G16 data set using the formula $\log \delta^{k}=c_{1} F^{k}+c_{2}$ (Fig. A1). Fig. A2 shows the resulting CMD for simulation 3 in Table A1.

\section{A2 Recovering the AMD}

We apply the inference process described in Sections 3 and 4 to the simulated populations using isochrone Grid B from Table 3. Fig. A3 shows the AMD for Simulations 1, 2 and 3. For Simulation 1 the left and central panels show that the 11 Gyr population is clearly separated from the older population. For Simulations 2 and 3 the contribution at $11.5 \mathrm{Gyr}$ is identified but the time resolution of Grid B $(0.5 \mathrm{Gyr})$ is not enough to assign the correct age to the younger population, which looks as part of the older population in our solution for $a_{A G E}$. However, for Simulation 3 the fact that the younger population has a different metallicity than the old population allows its clear identification in the 3D plot on the left hand side in Fig. A3. For these 3 simulations we infer the true $a_{Z}$ distributions. Simulations 4 and 5 are similar to Simulations 2 and 3 but the number of stars in the younger burst is reduced by more than $80 \%$. In Fig. A4 the young population is barely identified in $a_{A G E}$ and not at all in $a_{Z}$.

In Fig. A5 we show the AMD inferred for Simulation 3 when the photometric errors are increased to $\sqrt{\left(\sigma^{k}\right)^{2}+0.02^{2}}$ mag. The most obvious effect of increasing the errors in the CMD is to broaden the MSTO, the RGB and the HB (cf. right hand side and left hand

12 http://stev.oapd.inaf.it/cgi-bin/cmd 
Table A1. Simulation parameters. Columns 2, 3, 4 correspond to the metallicity, age and number of stars brighter than F475W=28.5 for the older population, respectively, while columns $5,6,7$ are the younger population counterparts.

\begin{tabular}{ccccccc}
\hline \multirow{2}{*}{ Simulation } & \multicolumn{3}{c}{ Old population } & \multicolumn{3}{c}{ Young population } \\
& $Z$ & AGE (Gyr) & $N_{\text {old }}$ & $Z$ & AGE (Gyr) & $N_{\text {young }}$ \\
\hline 1 & 0.0001 & $12.25,12,11.75$ & 4253 & 0.0001 & 11.0 & 1721 \\
\hline 2 & 0.0001 & $12.25,12,11.75$ & 4253 & 0.0001 & 11.5 & 1730 \\
\hline 3 & 0.0001 & $12.25,12,11.75$ & 4253 & 0.0002 & 11.5 & 1700 \\
\hline 4 & 0.0001 & $12.25,12,11.75$ & 4253 & 0.0001 & 11.5 & 292 \\
\hline 5 & 0.0001 & $12.25,12,11.75$ & 4253 & 0.0002 & 11.5 & 252 \\
\hline
\end{tabular}
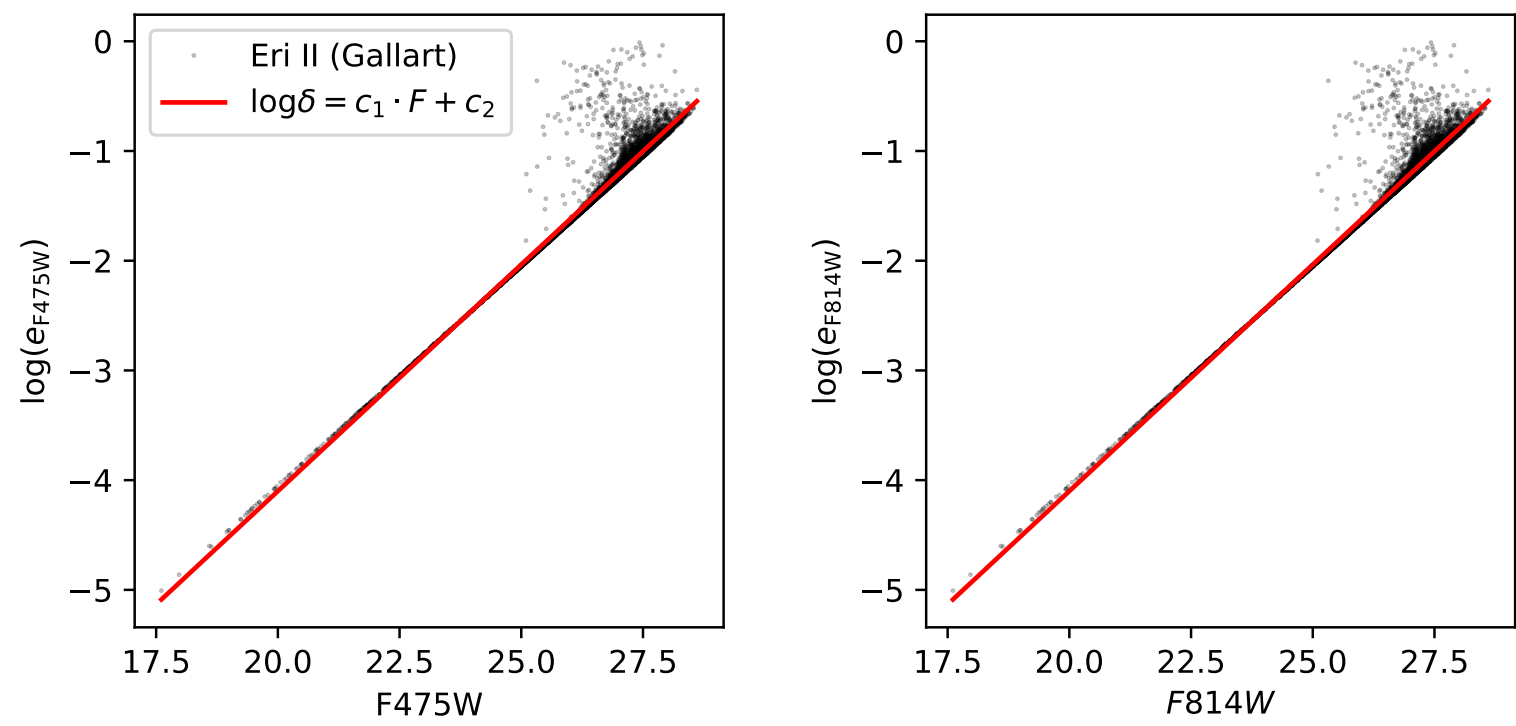

Figure A1. Photometric errors for the G16 data sets. (Left panel) F475W, (right panel) F814W. The coefficients of the best fit (red line) are $(c 1, c 2)=(0.4171,-12.8011)$ and $(0.4129,-12.3588)$ for $\delta_{F 475 W}$ and $\delta_{F 814 W}$, respectively.
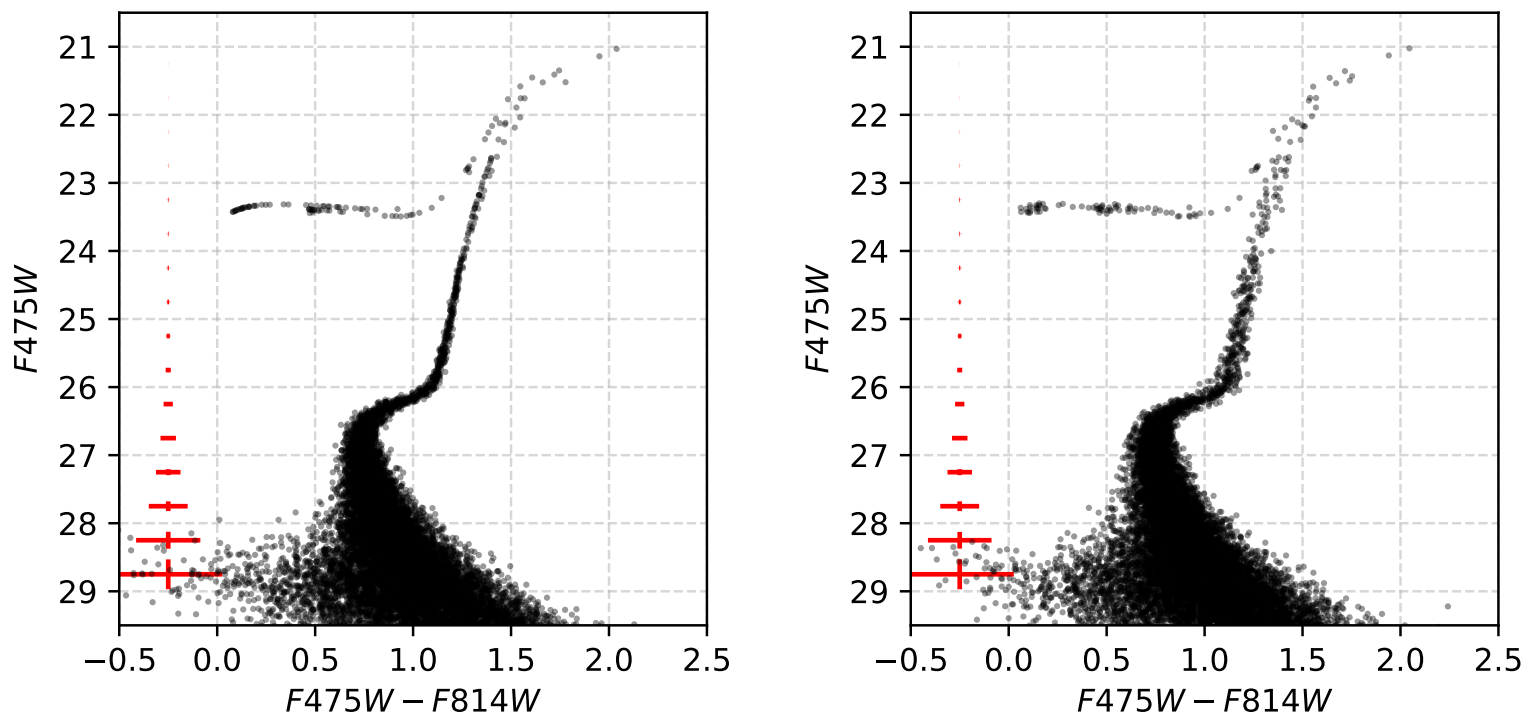

Figure A2. CMD for Simulation 3. (Left panel) Photometric errors from Fig. A1. (Right panel) Photometric errors increased by a constant value of 0.02 mag. 

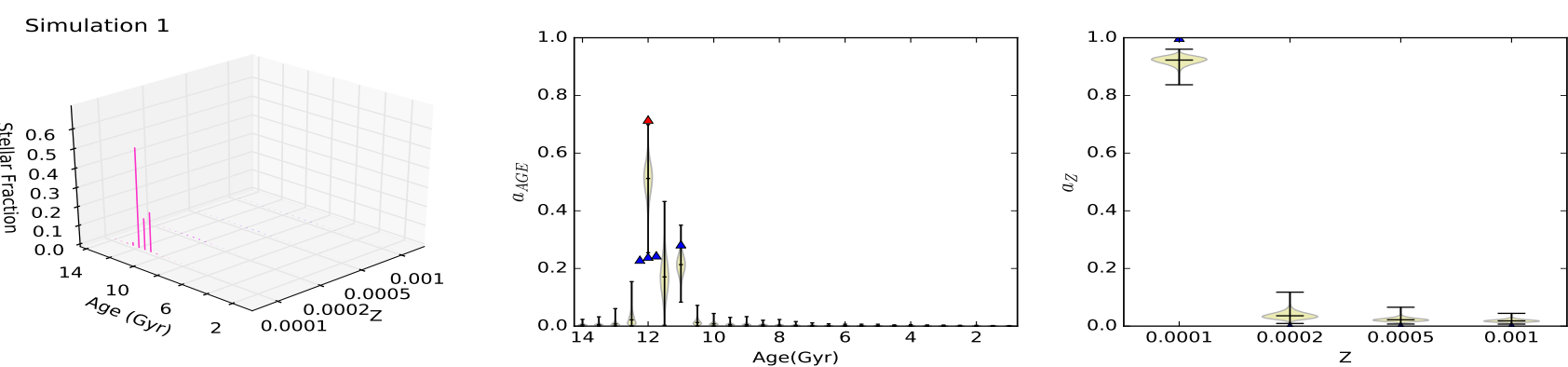

Simulation 2
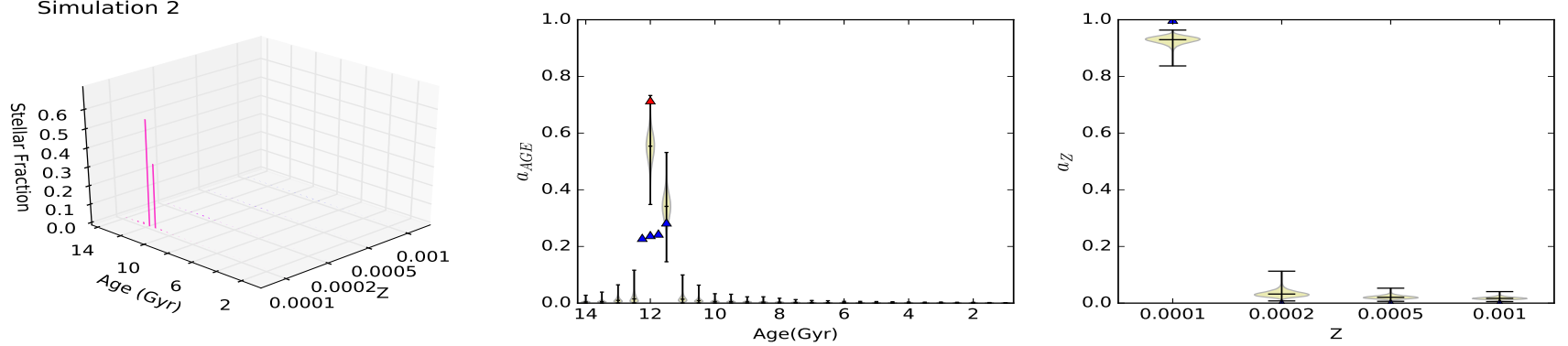

Simulation 3
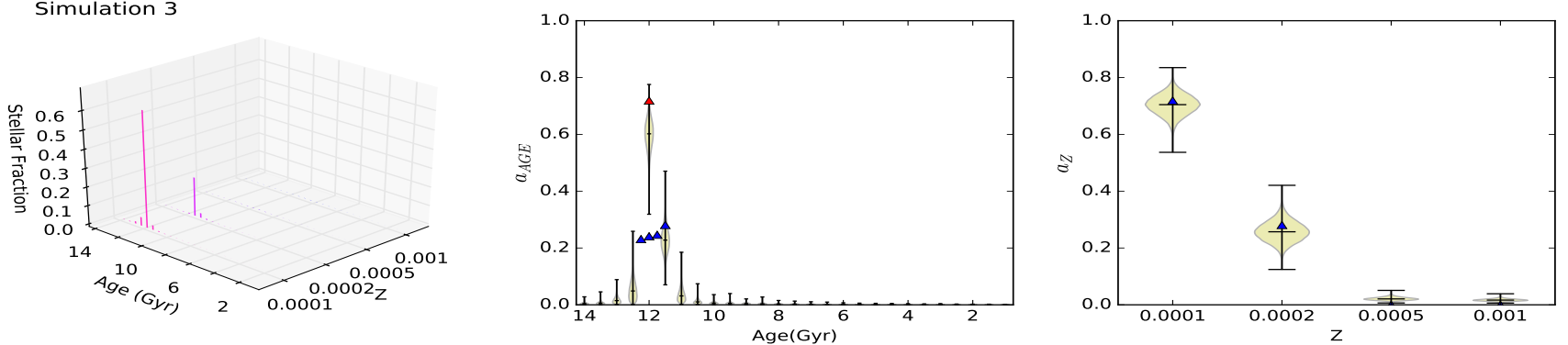

Figure A3. Inferred AMD for Simulations 1 (upper row), 2 (middle row) and 3 (bottom row), derived using isochrone Grid B (Table 3 ) and $\sigma_{i}^{k}=0.01$ mag. The blue triangles represent the input values of $a_{A G E}$ and the red triangle the sum of $a_{A G E}$ for the older population.
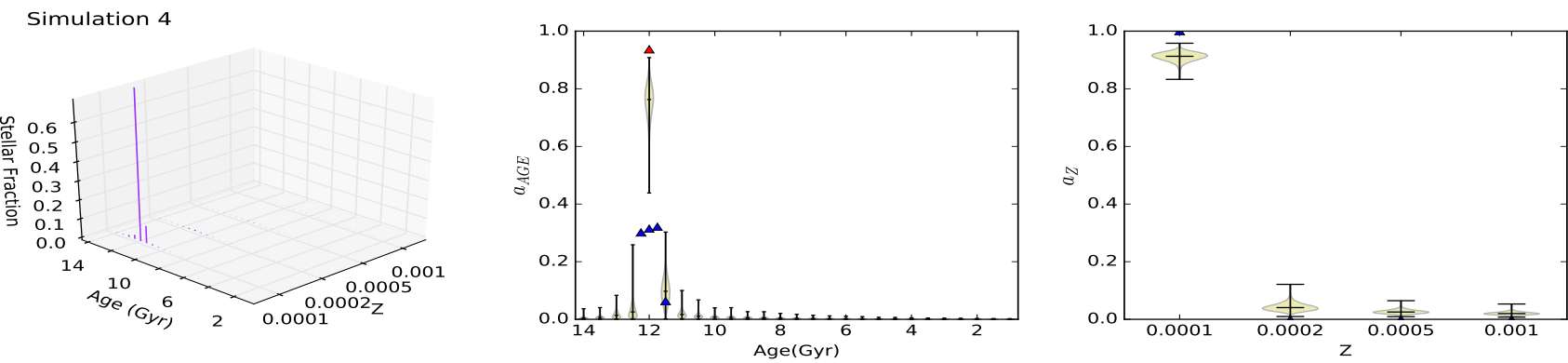

Simulation 5
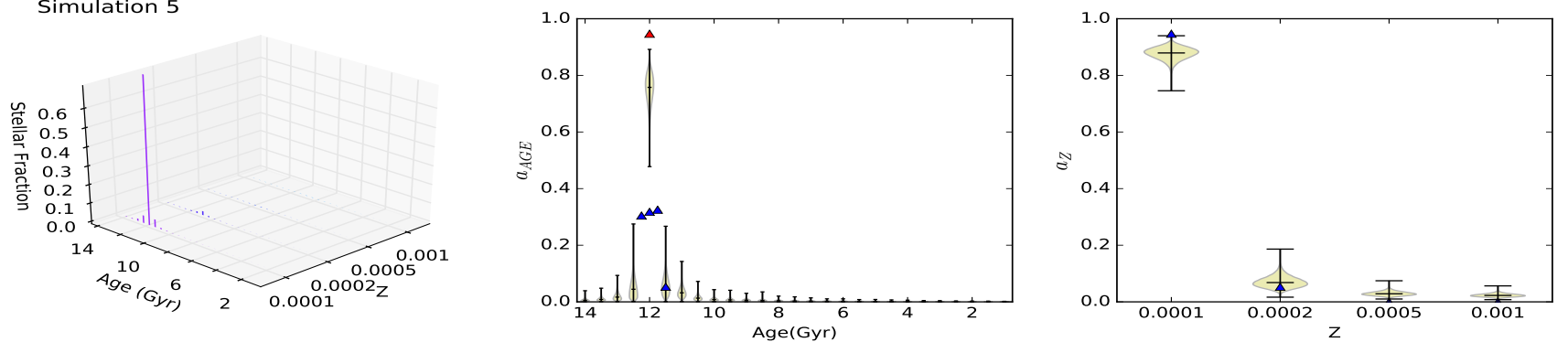

Figure A4. Inferred AMD for Simulations 4 (upper row) and 5 (bottom row), derived using isochrone Grid B (Table 3 ) and $\sigma_{i}^{k}=0.01$ mag. The blue triangles represent the input values of $a_{A G E}$ and the red triangle the sum of $a_{A G E}$ for the older population. 
Simulation 3

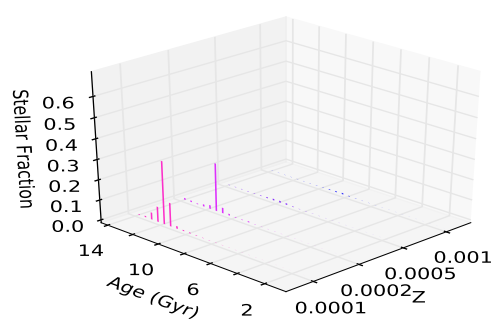

Simulation 3

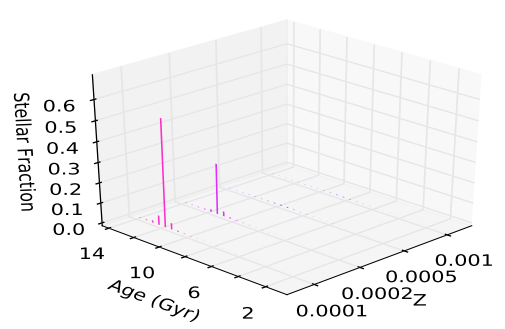

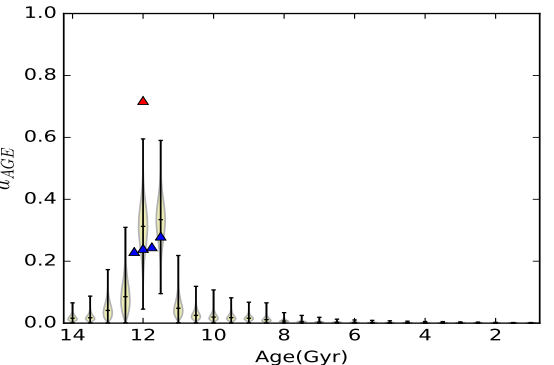
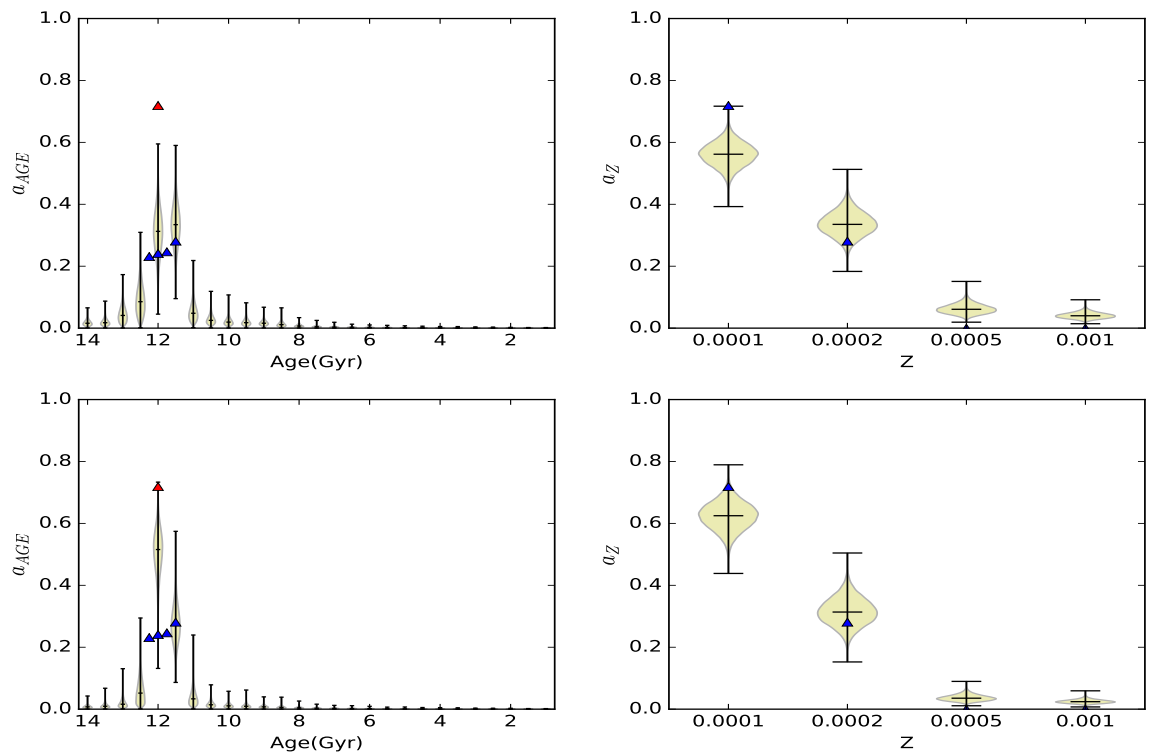

Figure A5. Inferred AMD for Simulation 3 with photometric errors increased by 0.02 mag derived using isochrone Grid B (Table 3 ). (Upper row) Results for $\sigma_{i}^{k}=0.01$ mag. (Lower row) Results for $\sigma_{i}^{k}=0.02 \mathrm{mag}$. The blue triangles represent the input values of $a_{A G E}$ and the red triangle the sum of $a_{A G E}$ for the older population.

side panels of Fig. A2). The upper row of Fig. A5 shows the AMD recovered using isochrone Grid $\mathrm{B}$ and $\sigma_{i}^{k}=0.01 \mathrm{mag}$, whereas the lower row corresponds to the AMD recovered using the same isochrone grid but $\sigma_{i}^{k}=0.02 \mathrm{mag}$. It is clear that the solution for $\sigma_{i}^{k}=0.02 \mathrm{mag}$ is closer to the true value, especially for $a_{A G E}$.

From this experiment we conclude that we can recover correctly the age of stellar populations of the same metallicity born in different star formation episodes separated in time by more than the time resolution of the isochrone grid in use. Nearly coeval populations of different metallicity can be characterized as long as the number of stars in the less massive population is significant. The size of the photometric errors included in the statistical model must resemble the true errors.

This paper has been typeset from a $\mathrm{T}_{\mathrm{E}} \mathrm{X} / \mathrm{L} \mathrm{T} \mathrm{T} \mathrm{X}$ file prepared by the author. 